\title{
ВАРИАНТЫ СУБОПТИМАЛЬНОГО СИНТЕЗА УПРАВЛЕНИИ МЕТОДОМ АГРЕГАЦИИ
}

Рассматривается задача управления нелинейной динамической системой с интегральным критерием качества. Исследуются различные способы агрегации системы порядка $n$ для приближенного решения задачи оптимального синтеза управлений. Выражения субоптимальных регуляторов определяются методом производящих функций Беллмана-Ляпунова из агрегированной системы. Предложены критерии оптимизации приближенных решений по вектору агрегации и примеры решения задач оптимальной стабилизации движений некоторых консервативных и $y$-автономных систем.

1. Рассмотрим уравнения возмущенных движений объекта

$$
\begin{aligned}
& x=X(t, x, u, c) \text { на } R=\left\{t \geqslant 0,\left|x^{1}\right|<\varrho\right\}, \\
& u \in U=\{u(t, x, c) \subset C(R \times A), u \in \Omega\},
\end{aligned}
$$

$x=\left(x_{j}, x_{k}\right)^{*}, \quad x^{1}=\left(x_{j}\right)^{*}, \quad x^{\prime}=\left(x_{i}\right)^{*}, \quad x^{\prime \prime}=\left(x_{v}\right)^{*}, \quad x^{(2)}=\left(x_{\beta}\right)^{*}, \quad u=\left(u_{\sigma}\right)^{*}$,

$x \equiv d x / d t, \Omega \subseteq E^{r}, \mathrm{Q}=\mathrm{const}, i=\overline{1, l} \leqslant m, j=\overline{1, m} \leqslant n, \beta=\overline{m+1, n}$,

$$
\sigma=\overline{1, r} \leqslant n,
$$

$v=\overline{l+1, m}$, const $=c=\left(c_{k}\right)^{*}, k \geqslant 1, E^{k} \supseteq A-$ область, открытая или замкнутая, где $X, U$ удовлетворяют на $R^{0}=R \backslash x^{\prime}=0$ условиям существования, единственности $x[t]$ и продолжаемости $x^{(2)}[t]$ для $t \rightarrow+\infty$ при $t, x \in R^{0}, c \in A$.

Пусть $(1.1)$ - система Ляпунова по $x^{1}$ : существует $u^{\prime}[V(t, x, c)] \in$ $\in U$, для которого (1.1) имеет $x^{1}$-определенно положительную (1.2) на $R$ функцию $V(t, x, c)$, строго убывающую вдоль любой полутраектории $x[t] \equiv x\left(t, t_{0}, x_{0}, c\right) \Subset R^{0}$ на интервале $\left[t_{0}, t^{+}\right)$ее определения в $R^{0}$ и, кроме того,

$$
\begin{gathered}
V\left(t, 0, x^{(2)}, c\right) \equiv 0, \quad V \geqslant V_{1}\left(x^{1}, c\right)>0 \text { на } \\
R \backslash x^{1}=0\left(V, V_{1} \subset C(R), V \subset C_{1}\left(R^{0}\right), \forall c \in A\right), \\
V \cdot \leqslant 0, t, x \in R^{0}, c \in A, R_{0}^{0} \cap Q^{+}=\varnothing \\
\left(R_{0}^{0}=R^{0} \cap\left\{V^{\cdot}=0\right\}, Q^{+}=\left\{x_{0}^{+} \mid x\left(t, t_{0}, x_{0}^{+}\right) \subseteq Q^{+}\right\}\right), \\
V \cdot<0 \text { на } R^{+} \equiv R^{0} \cap Q^{+} \quad\left(V\left(t_{0}, x_{0}\right)>V(t, x[t]), t_{0} \leqslant t \leqslant t^{+},\right. \\
\left.x^{\prime}\left(t_{0}\right) \equiv x_{0}^{\prime} \neq 0, \forall c \in A\right), \\
t^{+}=\infty, t_{1}, t^{+}=+\infty \text { для } x^{\prime}[t] \neq 0, t_{0} \leqslant t, t_{1}=\min t^{1} \leqslant \infty
\end{gathered}
$$

$$
\left(x^{\prime}\left[t^{1}\right]=0, V \cdot \equiv-W\right) \text {. }
$$


В случае inf $\delta^{1}\left(t_{0}, x_{0}{ }^{(2)}, c, \varepsilon\right)>0$ на $C^{0} \equiv\left\{0 \leqslant t \leqslant t^{0},\left|x_{0}{ }^{(2)}\right| \leqslant \delta_{0}^{(2)}\right\}$ для $\forall \varepsilon<\mathrm{g}, \forall c \in A, t_{0}, x_{0}^{(2)} \in C^{0}$ из $(1.2)$ находим такое $\delta_{0}{ }^{1}(\varepsilon)<\delta^{1}$, что $\left|x^{1}[t]\right|<\varepsilon$ при $t_{0} \leqslant t \leqslant t^{+}, \quad\left|x_{0}^{1}\right|<\delta_{0}^{1}\left(x_{0} \equiv x\left[t_{0}\right]\right)$. Обозначим $\alpha=$ $=\alpha(c)=\min V_{\mathrm{I}}$ на $\left|x^{1}\right|=\varrho$. В общем случае система $(1.1),(1.2)$ будет $\left|x^{1}[t]\right|<\mathrm{Q}$ для $t \in\left[t_{0}, t^{+}\right)$, если $t_{0}, x_{0}$ взяты в цилиндре

$C_{1}^{0}=\left\{0 \leqslant t_{0} \leqslant t^{0},\left|x_{0}^{1}\right| \leqslant \delta_{0}^{1}(\varrho),\left|x_{0}^{(2)}\right| \leqslant \delta_{0}^{(2)}(\varrho), x_{0}^{\prime} \neq 0\right\}\left(\delta_{0}^{1^{2}}+\delta_{0}^{(2)}<\varrho^{2}\right)$,

где $\alpha>v_{0}{ }^{1} \equiv \max V\left(t_{0}, x_{0}, c\right) \quad$ на $t_{0}, x_{0} \in C_{1}{ }^{*}=\bar{C}_{1}\left(t^{0}=\right.$ const $<\infty$, $c=$ fixconst). Тогда из продолжаемости $x[t]$ на $\left[t_{0}, t^{+}\right)$следует, что любое решение $(1.1)\left(u=u^{\prime}\right)$ с началом в $C_{1}{ }^{0}$ принадлежит либо классу $F=\{f[t]\}$, либо классу $D=\{d[t]\}$. Класс $F$ состоит из решений (1.1), примыкающих к $x^{\prime}=0: x^{\prime} \rightarrow 0$ при $t \rightarrow t_{1} \leqslant \infty$. Класс $D$ составляют решения (1.1), не примыкающие к $x^{\prime}=0$ за конечное или бесконечное время. Из выражений $(1.1)-(1.3)$ и определения для $\forall f[t], d[t]$ имеем

$$
\varrho>\left|x_{f}^{1}(\tau)\right|>0, t_{0} \leqslant \tau \leqslant t_{1} \leqslant \infty, \lim _{\tau \rightarrow t_{1}} x_{f}^{\prime}[\tau]=0,\left|x_{f}^{\prime}[\tau]\right|>0\left(\tau \in\left[t_{0}, t_{1}\right)\right),
$$

$$
x_{f}^{1}\left(t_{1}\right)=0 \text { при } v^{0}=0,0 \leqslant v^{0} \equiv \lim _{\tau \rightarrow t_{1}-0} v_{f}[\tau]<v_{0} \equiv v_{f}\left[t_{0}\right] \leqslant v_{0}^{1}<\alpha,
$$

$$
\mathrm{Q}>\left|x_{d}^{1}(\tau)\right|>0, t_{0} \leqslant \tau \leqslant \infty, \varlimsup_{\tau \rightarrow \infty}\left|x_{d}^{\prime}[\tau]\right|=r^{0},\left|x^{\prime}(\tau)\right|>0\left(\tau \geqslant t_{0}\right),
$$

$$
\begin{aligned}
\lim _{\tau \rightarrow \infty}\left|x_{d}^{\prime}(\tau)\right| \equiv & r_{0} \geqslant \inf _{\tau \geqslant t_{0}}\left|x_{d}^{\prime}(\tau)\right| \equiv \varrho_{0} \geqslant 0\left(r_{0}=0 \text { при } \varrho_{0}=0,\left|x^{1}\right| \geqslant\left|x^{\prime}\right|\right), \\
& 0<v^{0} \equiv \lim _{\tau \rightarrow \infty} v_{d}[\tau]<v_{0} \equiv v_{d}\left[t_{0}\right] \leqslant v_{0}^{1}<\alpha= \\
& =\min _{\left|x^{1}\right|=\rho} V_{1}(v[\tau]=V(\tau, x[\tau], c), c=\text { const } \in A)
\end{aligned}
$$

Используя прямой метод Ляпунова для траекторий $f, d$ с началом в $C_{1}{ }^{0}$, получим следующие критерии $x^{\prime}$-стабилизации регулятором $u^{\prime}$ решения $x \equiv 0$ системы $(1.1)(u \equiv 0)$.

Т е ор е а 1. Регулятор $u^{\prime}(t, x, c)$ системь Ляпунова (1.1), (1.2) реализует для значений $t_{0}, x\left(t_{0}\right)$ в (1.3) $x^{\prime}$-стабилизацию движения $x \equiv 0$ и устойчивость по его $x^{1}$-компоненте, если вдоль любой $d$-траектории (1.1) $\left(u=u^{\prime}\right)$ с началом в $C_{1}{ }^{0}$ выполняется неравенство

$$
v^{0}<\int_{t_{0}}^{\infty} w[\tau] d \tau\left(w[\tau]=W\left(\tau, x[\tau], c \mid u^{\prime}(\tau, x[\tau], c)\right)=-V \cdot[\tau]\right) .
$$

Доказ а тельство. Действительно, из (1.2)-(1.4) для $\forall \varepsilon \leqslant \mathrm{Q}$, $c \in A$ и $t_{0}, x_{0}{ }^{(2)} \in C^{0}$ имеем $x^{1}$-устойчивость решения $x \equiv 0$, где $\exists \delta_{0}^{1}(\varepsilon)$, и цилиндр $C_{\varepsilon}{ }^{0}=\left\{\left|x_{0}{ }^{0}\right| \leqslant \delta_{0}{ }^{1}(\varepsilon), x_{0}{ }^{\prime} \neq 0\right\} \times C^{0}$ начальных значений, для которых $\left|x^{\prime}[t]\right| \leqslant\left|x^{\prime}[t]\right|<\varepsilon$ при $t_{0} \leqslant t \leqslant t^{+}\left(t_{f}^{+}=t_{1}, t_{d}+=\infty\right)$. Кроме того, любое решение $x[t] \in F$ при $t_{0}, x_{0} \in C_{\varepsilon}{ }^{0}$. Допустим противное. Интегрируя уравнение $V^{*}=-W$ вдоль $d[t]$ в области $R^{0}$, из (1.5) получим неравенство $v^{0} \leqslant 0$, которое противоречит (1.4) по определению $d[t] \in D$. В силу примыкания всех решений к $x^{\prime}=0$ при $\forall t_{0}, x_{0} \in C_{\varepsilon}{ }^{0}$ $(\varepsilon \leqslant \mathrm{Q}, c \in A)$ для $C_{1}{ }^{0}$ имеем $x^{\prime}$-стабилизацию $x \equiv 0$ на интервале $t_{1}-t_{0} \leqslant \infty$. Теорема доказана.

3 а меч ание 1 . В (1.2) и (1.5) не требуются $\left[{ }^{1,2}\right]$ дифференцируемость $V$ на $x^{\prime}=0$, равномерность по $t, x^{(2)}$ условия $V \rightarrow 0$ при $x^{1} \rightarrow 0$, 
равенство $u^{\prime}(t, 0, c) \equiv 0, \quad x^{\prime}$-определенная положительность $W:$ $W\left(t, x, c \|^{\prime} V, u^{\prime}\right)=W \gg 0$. Назовем стабилизирующим на. $C_{1}{ }^{0}$ класс регуляторов $U^{\prime}=\left\{u^{\prime}(t, x, c) \mid u^{\prime} \in U\right\}$ системы (1.1), производящих $x^{1}$ устойчивую $x^{\prime}$-стабилизацию $x \equiv 0$ для $t_{1} \leqslant \infty, t_{0}, x_{0} \in C_{1}{ }^{0}$. Согласно теореме 1, класс $U^{\prime}$ для системы Ляпунова (1.1) определяется из условий $(1.2),(1.5)$ на функции $X, V$. Условия выбора стабилизирующих регуляторов упрощаются в леммах 1 и 2, где используются свойства решений дифференциальных неравенств и обозначения:

$$
\begin{gathered}
v=v(t, x, c) \geqslant v_{1}\left(x^{\prime}, c\right)>0,0<\left|x^{\prime}\right|<\mathrm{e},\left.v\right|_{x^{\prime}=0} \equiv \\
\equiv 0\left(v, v_{1} \subset C(R), v \subset C_{1}\left(R^{0}\right)\right),
\end{gathered}
$$

$v_{0}^{1}=\sup v(t, x, c)$ на $Q=\left\{R \cap V(t, x, c) \leqslant v_{0}^{1}\right\}\left(Q^{0}=Q x^{\prime}=0 \quad v_{0}^{1} \leqslant \infty\right)$, $v_{0}^{0}=\max _{C_{1^{*}}} v\left(t_{0}, x_{0}, c\right) \leqslant v_{0}^{1}, v_{0}^{1}=\max V\left(t_{0}, x_{0}, c\right)$ на $C_{1}^{*}=\bar{C}_{1}^{0}=C_{1}^{0} \cup x^{\prime}=0$,

$$
v_{0}=v\left[t_{0}\right], v_{0}=
$$

$=v\left[t_{0}\right]\left(0<V_{1}\left(x_{0}^{1}, c\right) \leqslant v_{0} \equiv V\left(t_{0}, x_{0}, c\right) \leqslant v_{0}^{1} \leqslant \alpha \equiv \min _{\left|x^{1}\right|=\rho} V_{1}, c=\right.$ fixconst $\left.\in A\right)$,

$\Lambda_{0}=\left\{0 \leqslant t_{0} \leqslant t^{0}, 0<v_{0}<v_{0}^{1}, 0<v_{0} \leqslant v_{0}^{0}\right\}, \quad \Lambda=\left\{t \geqslant 0,0<v<v_{0}^{1}, 0<v \leqslant v_{0}^{1}\right\}$.

Л ем а 1. Пусть для (1.1), (1.2), (1.6) на $R^{0}$ неравенства

$$
\begin{gathered}
v^{*} \leqslant-g_{1}(t, v, v, c), \\
v^{*} \leqslant-g_{2}(t, v, v, c)\left(g_{1}, g_{2} \subset C(\Lambda), g_{1}>0, v=v[t], \forall c \in A\right)
\end{gathered}
$$

в области $\Lambda$ не имеют решений, удовлетворяющих условиям

$$
\lim _{t \rightarrow \infty} v[t]>0, \varlimsup_{t \rightarrow \infty} v[t]>0, t_{0}, v_{0}, v_{0} \in \Lambda_{0}\left(v<<0 \text { на }\left[t_{0}, t^{+}\right)\right) .
$$

Тогда регулятор $u^{\prime}(t, x, c) \in U$ является стабилизирующиим на $C_{1}{ }^{0}$.

Дока зательство. Покажем, что $\forall x[t] \in F$ при $t_{0}, x_{0} \in C_{1}$ и условиях леммы. Допустим противное - на $\left[t_{0}, \infty\right)$ существует $d *[t]$ с началом в $C_{1}{ }^{0}$ и свойствами (1.4). Рассмотрим функции $v *[t]=$ $=v(t, d *[t], c), v \cdot[t]=V(t, d \cdot[t], c)$. При $t_{0} \leqslant t$ их значения лежат в $\Lambda_{0}, \Lambda$. Из (1.7) имеем оценки $v_{*}^{*} \leqslant-g_{1}\left(t, v_{*}, v_{*}, c\right), v_{*}^{*} \leqslant$

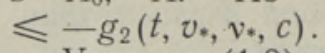

Условия (1.8) выполняются, так как примыкание $v *, v *$ к нулю при $t \rightarrow+\infty$ противоречит неравенствам $V \geqslant V_{1}, v \geqslant v_{1}$ и свойствам (1.4). Но так как (1.7) и (1.8) не имеют решения $v *[t], v *[t]$, то $D=\varnothing$ и все рассматриваемые $x[t] \in F$. Лемма доказана.

Рассмотрим ее модификацию в случае существования для системы $(1.1),(1.2),(1.6),(1.7)$ потенциала $P(v, v, c)$, удовлетворяющего в $\Gamma=\{v \geqslant 0, v \geqslant 0\}$ условиям

$P \geqslant P(0,0, c)=0, P>0, v>0, P \subset C(\Gamma)$, при $v \rightarrow \infty P \rightarrow \infty(\forall v>0, c \in A)$,

$$
\begin{gathered}
g_{2}^{\prime \prime}=g_{1}^{\prime} \frac{\partial P}{\partial v}+g^{\prime}{ }_{2} \frac{\partial P}{\partial v} \geqslant 0 \text { на } \Gamma^{0}=\{v>0, v>0\} \\
0 \leqslant \frac{\partial P}{\partial v} \subset C\left(\Gamma^{0}\right), 0<\frac{\partial P}{\partial v} \subset C\left(\Gamma^{0}\right) \\
v^{*} \leqslant-g^{\prime}{ }_{1}(t, v, v, c), v^{*} \leqslant-g_{2}^{\prime}(t, v, v, c) \text { на }
\end{gathered}
$$




$$
\Lambda^{0}=T \times \Gamma^{0}\left(0<g_{1}^{\prime}, g_{2}^{\prime} \subset C\left(\Lambda^{0}\right), T=[0, \infty)\right) .
$$

Из (1.9) следует, что для (1.7) $P-$ это функция Ляпунова вида

$$
\begin{aligned}
p^{*} \leqslant-g_{2}^{\prime \prime} \leqslant 0 & \text { на } \Lambda^{0}, \quad p[t] \equiv P(v[t], v[t], c) \leqslant p\left[t_{0}\right] \equiv \\
& \equiv p_{0}\left(p_{0} \geqslant p_{d}\left[t^{+}\right]=p_{d}^{+}>0\right),
\end{aligned}
$$

$$
p_{0}=P\left(v_{0}, v_{0}, c\right)>P\left(v_{0}, 0, c\right)>0 \quad v_{0}>v>0\left(\lim _{t \rightarrow t^{*}} v[t]=v^{0}<v_{0}, v_{0}=v\left[t_{0}\right]\right) .
$$

Из (1.9) и (1.10) заключаем, что неотрицательное решение дифференциальных неравенств (1.9) ограничено. При этом предельное множество траектории, соответствующей $d$-решению, не пусто и состоит из единственной точки $v^{0}>0, v_{*}{ }^{0}>0$, удовлетворяющей уравнению

$P\left(v^{0}, v_{*}^{0}, c\right)=p^{0}\left(v^{0}=\lim _{t \rightarrow \infty} v[t], 0<p^{0}=\lim _{t \rightarrow \infty} p[t], 0<v_{*}^{0}=\lim _{t \rightarrow \infty} v[t]\right)$,

$$
0<\psi\left(v^{0}, c\right) \equiv P\left(v^{0}, 0, c\right)<p^{0} \leqslant p[t] \leqslant p_{0}=p\left[t_{0}\right] \quad(\psi(v, c) \equiv P(v, 0, c)) .
$$

В силу (1.9) существует единственное решение $v^{*}=v^{*}(v, p, c)$ уравнения $(1.11), \quad$ где $0 \leqslant v^{*}(v, p) \subset C\left(\Gamma_{1}\right), \quad 0<v^{*} \subset C_{1}\left(\Gamma_{1}\right)^{0}, \quad \Gamma_{1}=$ $=\{v \geqslant 0, p \geqslant \psi\}, \Gamma_{1}{ }^{0}=\{v>0, p>\psi\}$. С учетом (1.11) из (1.9) и (1.10) подстановкой $v^{*}$ на $\Gamma_{1}^{0}$ находим

$$
\begin{gathered}
v \leqslant-g_{1}^{0}(t, v, p, c), p \leqslant-g_{2}^{0}(t, v, p, c) \quad\left(0<\psi\left(v^{0}, c\right)<p^{0} \leqslant p, 0<v^{0}<v\right), \\
g_{1}^{0}=g_{1}^{\prime}\left(t, v, v^{*}(v, p, c), c\right)>0, g_{2}^{0}=g_{2}^{\prime \prime}\left(t, v, v^{*}(v, p, c), c\right) \geqslant 0 \\
\left(g_{h} \subset C\left(\Gamma_{1}^{0}\right), h=\overline{1,2}\right) .
\end{gathered}
$$

Пусть существует интегрируемая $g[\tau]$, для которой имеем

$$
\begin{gathered}
G^{0} \equiv v^{0} g_{1}^{0}+p^{0} g_{2}^{0} \geqslant g[\tau] \equiv g\left(\tau, v_{0}, p_{0}, v^{0}, p^{0}, c\right)>0 \\
\left(v^{0} \leqslant v \leqslant v_{0}, \psi\left(v^{0}\right)<p^{0} \leqslant p \leqslant p_{0}\right),
\end{gathered}
$$

$\lim _{t \rightarrow+\infty} \int_{t_{0}}^{t} g[\tau] d \tau>s\left[t_{0}\right]-s[\infty] \quad\left(s=1 / 2\left(v^{2}+p^{2}\right),-s^{*} \geqslant G \equiv v g_{1}^{0}+p g_{2}^{0}>G^{0}\right)$.

Используя функцию $s(v, p)$ и (1.12), аналогично получаем результат:

Л ем м а 2. Регулятор $u^{\prime}(t, x, c)$ стабилизирует на $C_{1}{ }^{0}$ систему $(1.1)$, (1.2), если при $и=u^{\prime}$ она удовлетворяет условиям (1.9) $и$ (1.13).

3 амечание 2 . В леммах 1 и 2 нет условия $\operatorname{dim} x^{\prime}=\operatorname{dim} x^{1}$, требования $W \gg 0$ по $x^{\prime}\left[{ }^{2,3}\right]$ и равномерной ограниченности $v_{1}{ }^{*} \leqslant N_{1}{ }^{2}=$ $=$ const $>0$ для выбора $u^{\prime}\left(v_{1} \equiv\left|x^{\prime}\right|^{2}\right)\left[{ }^{3}\right]$. Отметим, что доказательство теоремы проходит, если $v_{1} \geqslant-N_{2}{ }^{2}=$ const $<0$ в точках $H$, где $v_{1}{ }^{\circ}>N_{1}{ }^{2}>0\left[{ }^{3}\right]$. Теорема в $\left[{ }^{3}\right]$ есть следствие леммы 2. Покажем, что при условиях $\left[{ }^{3}\right]$ имеем подслучай леммы 2 , при котором выполняются неравенства

$$
\begin{gathered}
W(t, x) \geqslant w\left(x^{\prime}\right) \geqslant g^{\prime}{ }_{1}\left(v_{1}\right), v_{1}>0, g^{\prime}{ }_{1}(0)=0, \int_{0}^{\infty} g^{\prime}{ }_{1}(\xi) d \xi=+\infty \\
\left(g^{\prime}{ }_{1} \subset C[0, \infty)\right), \\
v \leqslant-g_{1}^{\prime}, v \leqslant-g^{\prime}{ }_{2} \equiv N_{1}^{2}=\text { const, } P\left(v, v_{1}\right)=N_{1}^{2} v+\Phi^{-1}\left(v_{1}\right) \\
\left(v_{1} \equiv\left|x^{\prime}\right|^{2}, c=\text { fixconst }=c_{0} \in A\right),
\end{gathered}
$$




$$
\begin{gathered}
\Phi^{-1}\left(v_{1}\right) \equiv \int_{0}^{v_{1}} g^{\prime}{ }_{1}(\xi) d \xi, \quad g_{1}^{0}=g_{1}\left[\Phi\left(p-N_{1}^{2} v\right)\right], \quad g_{2}^{0}=g_{2}^{\prime \prime}=g_{1}^{\prime} \frac{\partial P}{\partial v}+g_{2}^{\prime} \frac{\partial P}{\partial v}=0 \\
p^{0}-P\left(v^{0}, 0\right)=\int_{0}^{v^{0}} g_{1}(\xi) d \xi>0, v_{*}^{0}>0,0<g_{1}^{*}=\min g_{1}^{0}(v, p) \quad \text { на } \\
G^{0}=v^{0} g_{1}^{0}>v^{0} g_{1}^{*} \equiv g[\tau] \equiv \text { const }>0, s\left[t_{0}\right]-s[\infty]<\int_{0}^{\infty} g d \tau=\infty \\
\quad\left(\Phi \left[\Phi^{0} \leqslant p \leqslant p_{0},\right.\right. \\
s=1 / t_{2}\left(v^{2}+p^{2}\right), s\left[t_{0}\right]=1 / 2\left(v_{0}^{2}+p_{0}^{2}\right), s[\infty]=1 / 2\left(v^{0^{2}}+p^{0^{2}}\right) .
\end{gathered}
$$

В силу (1.14) для теоремы из $\left[{ }^{3}\right]$ выполняются все условия леммы 2. Заменой $W>0$ на $W \geqslant 0$ (при $\left.\operatorname{dim} x^{\prime}<\operatorname{dim} x^{1}\right), g_{1}>0$ на $g_{1} \geqslant 0$ и $P(v, v, c)$ на $P(t, v, v, c)$ при надлежащей модификации условий лемм 1 и 2 и теоремы 1 можно ослабить (усложняя) ограничения, задающие для (1.1) класс $U^{\prime}$. Для построения $U^{\prime}$ возможно использовать $\Delta x^{\prime}$ и условия, дающие равномерную непрерывность, липшицевость, равномерную устойчивость по $t_{0}$, а также ограниченность изменения $V_{t_{0}}^{t^{+}} x^{\prime}$.

2. Поставим задачу оптимизации (1.1) при $u=u_{0}^{\prime}$ по критерию

$$
\begin{gathered}
I(t, x, c \mid u)=\int_{t}^{t_{1}} L(\tau, x, u) d \tau, \min _{u^{\prime} \in U^{\prime}} I=I^{0}(t, x, c)=I\left(t, x, c \mid u_{0}^{\prime}\right), \\
0 \leqslant L(t, x, u) \subset C\left(T \times E^{n} \times \Omega\right), L \subset C_{1}\left(T \times E_{0}^{n} \times \Omega_{0}\right), \\
E_{0}^{n}=E^{n} \backslash x^{\prime}=0, \quad \Omega_{0}=\Omega \backslash 0,
\end{gathered}
$$$$
u, u^{\prime}, u_{0}^{\prime} \in U=\left\{u(t, x, c) \subset C, u \in \Omega \subseteq E^{r}\right\}, c=\text { const } \subseteq A,
$$$$
\infty \geqslant t_{1}=\min t^{1}: x^{\prime}\left[t^{1}\right]=0 \text {. }
$$

Ее решение - минимум интеграла, и $u$, стабилизирующий на $C_{1}{ }^{0}$, обозначим через $I^{0}, u_{0}^{\prime}$. Введем переменные агрегации $\xi_{i}=\xi_{i}(t, x, a)$ преобразованием

$$
y_{1}=v(t, x, a), y_{2}=v(t, x, a), z_{\beta}=z_{\beta}(t, x, a), \xi=\left(y_{1}, y_{2}, z_{\beta}\right)^{*},
$$

$y=\left(y_{\alpha}\right)^{*}, z=\left(z_{\beta}\right)^{*}, a=\left(a_{k}\right)^{*}=\mathrm{const} \in A \subseteq E^{k}, \bar{S}(0, \delta) \subseteq A(\delta>0, k \geqslant 1)$,

$$
\inf _{T \times E^{n},\left|x^{\prime}\right| \geqslant \varepsilon} v(t, x, a)>0, \forall \varepsilon>0, z_{\beta}(t, 0, a) \equiv 0
$$

$$
(i=\overline{1, n}, \alpha=\overline{1,2}, \beta=\overline{1, n-2}),
$$

обратимым на

$$
R^{0} \leftrightarrow D^{0}, C_{1}^{0} \leftrightarrow D_{1}^{0}=\left\{0 \leqslant t_{0} \leqslant t^{0}, 0<v \leqslant v_{0}^{1}, 0<v \leqslant v_{0}^{0}, z_{\beta}^{-} \leqslant z_{\beta} \leqslant z_{\beta}^{+}\right\},
$$

$T=\{t \geqslant 0\}$ при $\forall a \in A$, где $z_{\beta}^{-}=\inf z_{\beta}, z_{\beta}^{+}=\sup z_{\beta}$ на $R$,

$$
\begin{gathered}
\Gamma_{-}^{4}=\left\{z_{\beta}^{-} \leqslant z_{\beta} \leqslant z_{\beta}^{+}\right\}, P_{1}^{0}=\left\{0 \leqslant t_{0} \leqslant t^{0}, 0<v \leqslant v_{0}^{1}, 0<v \leqslant v_{0}^{0}\right\}, \\
P=T \times\{y(R)\} \equiv P^{0} \cup y_{2}=0 .
\end{gathered}
$$

Здесь $D, D^{0}, D_{1}{ }^{0}$ - образы областей $R, R^{0}, C_{1}{ }^{0}, z_{\beta} \subset C(T \times R \times A)$, 
$z_{\beta} \subset C_{1}\left(T \times F^{\circ} \times A\right), D=D(a)=\xi(R), z_{\beta}$ - переменные типа углов; $v, v$ - переменные типа действия в смысле $(1.2),(1.6)$ при $c=a$, где $a$ - вектор агрегации. В новых координатах имеем эквивалентные $(1.1),(2.1)$ модель и задачу

$\xi=F(t, \xi, u, a), y^{*}=Y(t, y, z, u, a), z^{*}=Z(t, y, z, u, a) \quad\left(c=c_{0} \in A\right)$,

$$
\begin{gathered}
J=\int_{t}^{t_{1}} L^{\prime}(\tau, y, z, u, a) d \tau \quad\left(L^{\prime}=L(t, x(t, \xi, a), u) \geqslant 0\right), \\
I\left(t, x, c_{0} \mid u^{\prime}\right)=J\left(t, \xi, a \mid u^{\prime \prime}\right), u^{\prime \prime}=u^{\prime \prime}(t, \xi, a)=u^{\prime}\left(t, x(t, \xi, a), c_{0}\right), \\
t^{\prime}{ }_{1}=t_{1}=\min t^{1}: y_{2}\left[t^{1}\right]=0, u, u^{\prime \prime}, u_{0}^{\prime \prime} \in U=\{u(t, \xi, a) \subset C, u \in \Omega\},
\end{gathered}
$$

$\min _{u^{\prime \prime} \in U^{\prime \prime}} J=J^{0}(t, \xi, a)=J\left(t, \xi, a \mid u_{0}^{\prime \prime}\right) \quad\left(\forall a \in A,\left(c_{k}^{0}\right)^{*}=c_{0}=\right.$ fixconst $\left.\in A\right)$,

$$
\begin{gathered}
F(t, 0,0, a) \equiv 0, F=X(\xi), Y=X(y), Z=X(z), \\
X(f)=\frac{\partial f}{\partial t}+X_{s} \frac{\partial f}{\partial x_{s}}(s=\overrightarrow{1, n}) .
\end{gathered}
$$

С учетом (1.1), (1.2), (2.2) решения (2.3) при $t_{0}, \xi\left[t_{0}\right] \equiv \xi_{0} \in D_{1}^{0}(a)$, $u^{\prime \prime} \in U$ существуют, единственны и $z$-продолжаемы на $D^{0}(a)$, где $a=$ $=c=$ const, $\quad F \in C_{1}\left(D^{0} \times \Omega_{0} \times A\right)$. Обозначим через $U^{\prime \prime}=$ $=\left\{u^{\prime \prime}(t, \xi, a) \mid u^{\prime \prime} \in U\right\}$ класс регуляторов $\left\{u^{\prime \prime}\right\}$ системы (2.3), производящих $y_{1}$-устойчивую $y_{2}$-стабилизацию $\xi \equiv 0$ для $t_{1} \leqslant \infty, t_{0}, \xi_{0} \in D_{1}{ }^{0}$. Для $\forall a \in A, \quad c=c_{0} \quad$ имеем $\quad u^{\prime \prime}(t, \xi, a)=u^{\prime \prime}(t, \xi(t, x, a), a)=u^{\prime}\left(t, x, c_{0}(a)\right)$, $U^{\prime \prime} \leftrightarrow U^{\prime}$. Будем искать приближенные (субоптимальные) решения задачи (2.1) $y$-агрегацией $\left[{ }^{4-6}\right]$ и методом производящих функций $S(t, \xi, a)$ Беллмана-Ляпунова, используя эквивалентность оптимальных систем $(1.1),(2.1)$ и (2.3). Ниже выбор вектора агрегации $a$ подчиним критериям оптимальности $f(a)$. Возможны различные варианты агрегации $\left[{ }^{4-7}\right]$, соответствующие выбору -аппроксимации исходной модели, ограничений и критерия (композиционная агрегация) или погружению оптимизируемой системы в класс, порожденный априорными свойствами условного, геометрического и физического характера для ее $z$-подсистемы (экстремальная агрегация). Эффективность используемого способа построения агрегированной системы (модели и критерия) определяется типом модели, целями оптимизации и методом исследования. В качестве примера рассмотрим два варианта композиционной агрегации.

2.1. Вариант обращения, связанны й со сдвигом ядр а функционала. Агрегированную систему, соответствующую (2.3), введем заменой

$$
J_{1}^{+}=\int_{t}^{t^{+}} L_{1}^{+} d \tau, L_{1}^{+}=L_{1}^{\prime}+\Delta L_{1}^{\prime}\left(\Delta L_{1}^{\prime} \equiv L_{1}^{*}(t, \xi, a)=-B^{\prime}\left[S_{1}^{+}, u^{+}\right]\right),
$$

$\left.B^{\prime}\left[S, u^{0}\right] \equiv S \cdot\right|_{u=u^{0}}+L^{\prime}\left(u^{0}\right) \quad\left(\frac{\partial S}{\partial t}=p_{0}, \frac{\partial S}{\partial y_{\alpha}}=p_{\alpha}, p=\left(p_{\alpha}\right)^{*}, \psi=\left(\frac{\partial S}{\partial z_{\beta}}\right)^{*}\right)$,

где модель (2.3) сохраняется, а функция $S_{1}+(t, \xi, a)$, производящая регулятор $u^{+}\left[S_{1}^{+}\right] \in U$, удовлетворяет на $D^{0}$ для $(2.3)$ условиям

$$
\begin{gathered}
S_{1}^{+}\left(t, y_{1}, 0, z, a\right) \equiv 0, S_{1}^{+}(t, \xi, a) \geqslant 0,\left.\frac{d S_{1}^{+}}{d t}\right|_{u=u^{+}} \leqslant L_{1}^{\prime}(u)-L_{1}^{\prime}\left(u^{+}\right) \\
\left(L_{1}^{\prime} \equiv L^{\prime} \geqslant 0, L_{1}^{+}\left(u^{+}\right) \geqslant 0\right),
\end{gathered}
$$




$$
B^{\prime}\left[S_{1}^{+}, u\right] \geqslant B^{\prime}\left[S_{1}^{+}, u^{+}\right], u, u^{+} \in U .
$$

Пусть $u^{+}(t, \xi, a)$ - регулятор, стабилизирующий (2.3) на $D_{1}{ }^{0}$ в смысле п. 1. В частности, $u^{+} \in U^{\prime \prime}$, если в переменных $t, x$ этот регулятор и система (2.3) удовлетворяют теореме 2 или леммам 1 и 2 п. 1. Условия стабилизируемости в $t$, ६ получаются заменой $x^{(1)}, x^{\prime}, x^{(2)}, c=c_{0}$ соответственно на $y, y_{2}, z, a$.

Т ео р е м а 2. Допустимый регулятор $u^{+}(t, \xi, a)$ - оптимальный по $J_{1}{ }^{+}$, если выполнены неравенства (2.5) $и u^{+} \in U^{\prime \prime}$.

Доказательство. Из $(2.4),(2.5)$ следует для $\forall u, u^{+} \in U$ оценка

$$
B\left[S_{1}^{+}, u\right] \geqslant\left. B\left[S_{1}^{+}, u^{+}\right] \equiv \frac{d S_{1}^{+}}{d t}\right|_{u=u^{+}}+L_{1}^{+}\left(u^{+}\right)=0\left(t, \xi, a \in D^{0} \times A\right) .
$$

Интегрируя (2.6) вдоль траектории (2.3) при $u=u^{\prime \prime} \in U^{\prime \prime}, t_{0}, \xi_{0} \in D_{1}{ }^{0}$, с учетом $u^{+} \in U^{\prime \prime}$ и $(2.5)$ получим неравенства, доказывающие теорему:

$$
\min _{u \in U^{\prime \prime}} \int_{t}^{t_{1}^{+}} L_{1}^{+} d \tau=S_{1}^{+}(t, \xi, a) \leqslant \int_{t}^{t_{*}^{*}} L_{1}^{+} d \tau .
$$

Из (2.7) стабилизирующий $(2.3)$ на $D_{1}{ }^{0}$ регулятор $u^{+}(t, \xi, a)-$ субоптимальный по $J_{1}$ :

$J_{1}^{0}(t, \xi, a) \leqslant J_{1}\left(t, \xi, a \mid u^{+}\right) \leqslant S_{1}^{+}(t, \xi, a)$ при $B^{\prime}\left[S_{1}^{+}, u^{+}\right] \leqslant 0 \quad\left(t, \xi, a \in D^{0} \times A\right)$,

$$
S_{1}^{+}(t, \xi, a) \leqslant J_{1}^{0}(t, \xi, a) \leqslant J_{1}\left(t, \xi, a \mid u^{+}\right) \quad\left(B^{\prime}\left[S_{1}^{+}, u_{1}^{0}\right] \geqslant 0, J_{1}^{0}=J_{1}\left(u_{1}^{0}\right)\right) .
$$

Теорема 2 - это модификация в целях агрегации результатов $\left[{ }^{2,3,7,8}\right]$, полученных для решения проблемы обращения при более сильных предположениях. Приближенное решение $S_{1}+(a), J_{1}+(a), u^{+}(a)$ задачи $(2.3)$ экстремизируем по параметру $a$. Для простоты критерием оптимальности вектора агрегации $a$ * изберем для $\forall a, a_{*} \in A$ условие минимума $f(a.) \leqslant f(a)$ функционалов $f=f_{j}^{\alpha}(a)$ вида

$$
\begin{gathered}
f_{1}^{\alpha}(a)=\int_{C_{1}^{\prime 0}} f_{0}\left(t, x, a \mid V^{\alpha}\right) d t \prod_{s=1}^{n} d x_{s}\left(f_{0} \subset C\left(C_{1}^{\prime} \times A\right), C_{1}^{\prime 0} \subseteq C_{1}^{0}, \alpha=1,2\right), \quad(2.9) \\
V_{+}^{1} \equiv S_{1}^{+}(t, \xi(t, x, a), a), V_{+}^{(2)} \equiv J_{1}^{+}(t, \xi(t, x, a), a)\left(j=\overline{1,4}, J_{1}^{+} \equiv J_{1}\left(t, \xi, a \mid u^{+}\right)\right), \\
f_{2}^{1}(a)=V_{+}^{1}(t, x, a), f_{2}^{(2)}(a)=V_{+}^{(2)}(t, x, a)\left(\left(t, x_{s}\right)^{*}=\text { fixconst, } s=\overline{1, n}\right), \\
f_{3}^{(\alpha)}(a)=\underset{x^{\prime}}{\operatorname{div}} g^{\alpha}(t, x, a)=\sum_{i=1}^{l} \partial g_{i}^{\alpha} / \partial x_{i}\left(g^{\alpha} \cdot x^{\prime} \equiv f_{2}^{\alpha}, g^{\alpha}=\left(g_{i}^{\alpha}\right)^{*},\left.f_{2}^{\alpha}\right|_{x^{\prime}=0} \equiv 0\right), \\
f_{4}^{(\alpha)}(a)=\left|B\left[f_{2}^{\alpha}, u_{1}^{+}\right]\right|\left(u_{1}^{+}=u^{+}(t, \xi(t, x, a), a), B\left[f, u_{0}\right]=\left.\frac{d f}{d t}\right|_{u=u_{0}}+L_{1}\left(u_{0}\right)\right) .
\end{gathered}
$$

По теореме о среднем для $\forall t, x \in C_{1}^{10}$ из $f_{1}^{\alpha}$ получаем $f_{2}^{\alpha}$ надлежащим выбором $f_{0}$. В случае неквадратичных $V_{+}{ }^{\alpha}$ на $x^{\prime}$ показатель $f_{3}{ }^{\alpha}-$ аналог вероятностного критерия $\left[{ }^{5,9}\right]$ типа $\operatorname{tr}$. Невязка $f_{4}{ }^{\alpha}$ характеризует для $V_{+}{ }^{1}, V_{+}{ }^{(2)}$ локальную близость оптимального $u_{1}^{0}$ и субоптимального $u_{1}{ }^{+}$ регуляторов. Не требующее интеграции вдоль (1.1) при $u=u_{1}{ }^{+}$определение $f_{j}{ }^{1}$ проще определения $f_{j}^{(2)}$. Внутренний вектор $a_{*}^{\prime}$ агрегации $a *^{\prime}=a$, минимизирующий $f$, удовлетворяет уравнению 


$$
\partial f / \partial a_{*}=0\left(f=f_{j}^{\alpha}(a), a^{\prime} *=\left(a_{k}^{\prime}\right)^{*} \in i(A), k \geqslant 1\right) .
$$

Единственное решение (2.10) будет оптимальным вектором агрегации.

2.2. В торой в ари ант. Рассмотрим на $D \times A$ класс непрерывных вектор-функций $\Gamma_{1}=\{\gamma(t, y, a)\}$, непрерывно дифференцируемых вне $y_{2}=0$, со значениями в $\Gamma_{-}^{+}$. Подстановкой $z=\gamma_{*} \in \Gamma_{1}$, где $\Gamma_{-}^{+}=$ $=\left\{z_{\beta}-\leqslant z_{\beta} \leqslant z_{\beta}+\right\}$, в (2.3) получим агрегированную [4,5] систему

$$
y=Y \cdot(t, y, u \mid a), J_{2}^{*}=\int_{t}^{t_{*}} L \cdot(\tau, y, u \mid a) d \tau\left(t_{*}=\min t^{1}: y_{2}\left[t^{1}\right]=0\right) \text {, }
$$

$$
Y * \equiv Y(t, y, \gamma \cdot(\tau, y, a), u, a) \subset C_{1}\left(D^{0} \times A\right), Y=\left(Y_{1}, Y_{2}\right) *\left(D^{0}=D y_{2}=0\right),
$$

$$
\begin{array}{r}
0 \leqslant L_{*} \equiv L_{2}^{\prime}\left(t, y, \gamma_{*}(t, y, a), u, a\right) \subset C\left(D \times \Omega^{*} \times A\right), \\
L * \subset C_{1}\left(D^{0} \times \Omega_{0}^{*} \times A\right), L_{2}^{\prime}=L^{\prime},
\end{array}
$$

$$
u \in U_{*}^{\prime \prime}=\left\{u(t, y, a) \subset C\left(D^{0} \times A\right), u \in \Omega^{*}\right\}\left(\Omega^{*}=\left.\Omega\right|_{z=\gamma_{*}}, \Omega_{0}^{*}=\Omega^{*} \backslash u=0\right) .
$$

Здесь $U:^{\prime \prime}-$ класс стабилизирующих $(2.11)$ на $y \in D_{1}{ }^{0}$ регуляторов со значениями в образе $\Omega$. области $\Omega$ при композиции $z=\gamma_{*} ; y \in D^{0}$ на $\left[t_{0}, t_{*}\right)$ для $\forall u \in U *^{\prime \prime}$. Минимум $S_{2}{ }^{*}(t, y, a)$ функционала $J_{2}{ }^{*}$ на $U_{*}^{\prime \prime}-$ критерий оптимальности $u_{*}{ }^{0} \in U_{*}{ }^{\prime \prime}, S_{2}{ }^{*}=J_{2}\left[u_{*}{ }^{0}\right]$.

Пусть на $D^{0} \times A$ для $(2.11)$ известны $S_{2}{ }^{*}, u_{*}{ }^{0} \in U *^{\prime \prime}$, удовлетворяющие условиям

$$
\begin{gathered}
\left.S_{2}^{*}\right|_{y_{2}=0} \equiv 0, \mathrm{~S}_{2}^{*} \geqslant 0, B^{*}\left[S_{2}^{*}, u\right] \geqslant B^{*}\left[S_{2}^{*}, u_{*}^{0}\right]=0\left(u, u_{*}^{0} \in U_{*}^{\prime \prime}\right), \\
B^{*}\left[f, u^{0}\right] \equiv(d f / d t)_{u=u^{0}+L *\left(u^{0}\right)\left(f-=\partial f / \partial t+Y_{*} \cdot \partial f / \partial y\right),},
\end{gathered}
$$

где $u_{*}{ }^{0}$ - регулятор, стабилизирующий $(2.3)$ на $D_{1}{ }^{0}$, т. е. $u^{0} \in U^{\prime \prime}$. Из $(2.11),(2.12)$ находим, что $u_{*}, S_{2}^{*}$ - решение оптимальной задачи для $\gamma$ *-агрегированной системы (2.3), одновременно стабилизирующее субоптимальное для $(2.3)$ с $L^{\prime}=L_{2}^{\prime}$ в силу соотношений

$\min J_{2}^{*}=S_{2}^{*}(t, y(t, x, a), a) \equiv V_{*}^{1}, I_{2}^{0}(t, x) \leqslant J_{2}\left(t, \xi(t, x, a), a \mid u_{*}^{0^{\prime}}\right) \equiv V_{*}^{(2)}$. $u \in U^{\prime \prime}$.

Используя обозначения $(2.13)$ и замену $V_{+}{ }^{\alpha}$ на $V_{*}{ }^{\alpha}$ из $(2.9)$, получим критерии $a$-оптимальности. В отличие от варианта 2.1 в варианте композиционной агрегации 2.2 одновременно требуются включение $u{ }^{0} \in U^{\prime \prime}, u_{*}{ }^{0} \in U_{*}{ }^{\prime \prime}$ и нахождение решения уравнения Беллмана-Якоби со свойствами (2.12). При интерпретации $z=\gamma *(t, y, a)$ как связи на модель (2.3) в общем случае получаем дополнительное ограничение на $u$, не налагаемое в п. 2.1. В этом смысле способ агрегирования 2.2 грубее способа 2.1. Преимущество варианта 2.2 перед 2.1 состоит в $y$-автономности регулятора $u_{*}{ }^{0}$. Случай линейного объекта (1.1) с квадратичным критерием (2.1) подробно рассмотрен в $[5,10]$. В силу различия исходных постановок сравнение эффективности вариантов $2.1,2.2,2.3$ и их комбинирование здесь не проводятся.

2.3. Вариант экстремальной агрегация. Пусть для $z$ компоненты решения (2.3) на отрезке $\left[t_{0}, t_{1}\right]$ при $\forall u^{\prime \prime} \in U^{\prime \prime}$ и $\forall t_{0}$, $\xi_{0} \in D_{1}{ }^{0}, a, c_{0} \in A$ известна оценка Г-области достижимости по $z$ вида

$$
\begin{gathered}
\gamma_{\beta}^{0}(t, y[t], a) \leqslant z_{\beta}[t] \leqslant \gamma_{\beta}^{1}(t, y[t], a) \\
\left(\gamma^{\alpha} \in \Gamma_{1}, t_{0} \leqslant t \leqslant t_{1}, u=u^{\prime \prime} \in U^{\prime \prime}, \quad \alpha=0,1\right),
\end{gathered}
$$


$z \in \Gamma=\left\{z \mid \gamma_{\beta}^{0}(t, y, a) \leqslant z_{\beta} \leqslant \gamma_{\beta}^{1}(t, y, a)\right\}\left(\gamma_{\beta}^{0}\left(t_{0}, y_{0}, a\right) \leqslant z_{\beta}\left[t_{0}\right] \leqslant\right.$

$$
\leqslant \gamma_{\beta}^{1}\left(t_{0}, y_{0}, a\right), \beta=\overline{1, n-2)} \text {. }
$$

Для (2.3) введем две агрегированные системы $\Sigma_{0}, \Sigma_{1}$ с достаточно гладкими критериями $S_{3}{ }^{0}, S_{3}{ }^{1}$ их оптимальности

$y=Y(t, y, v, a), v=\left(z_{\beta}, u_{\sigma}\right)^{*} \in M \equiv G \times U, z \in G \equiv\left\{\gamma(t, y, a) \in \Gamma_{1}, \gamma \in \Gamma\right\}$,

$S_{3}^{0}(t, y, a)=\min _{z \in G} \min _{u \in U} J_{3}=\min _{v \in M} J_{3}\left(J_{3}=\int_{t}^{t_{1}} L^{\prime}{ }_{3} d \tau, L_{3}^{\prime}=L^{\prime}, u=\left(u_{\sigma}\right)^{*}, \quad \sigma=\overline{1, r}\right)$, $S_{3}^{1}(t, y, a)=\max _{z \in G} \min _{u \in U} J_{3}\left(t_{1}=\min t^{1}: y_{2}\left[t^{1}\right]=0, \quad U=\{u(t, \xi, a) \subset C, u \in \Omega\}\right)$.

В силу (2.14) оптимальная модель (2.3) содержится в объекте (2.15)модель систем $\Sigma_{0}, \Sigma_{1}$. Обозначим оптимальные стабилизирующие на $P_{1}{ }^{0}$ регуляторы систем $\Sigma_{0}, \Sigma_{1}$ через $v_{3}{ }^{0}, v_{3}{ }^{1}$. Для них функции $S_{3}{ }^{0}, S_{3}{ }^{1}$ удовлетворяют в области $P \times A$ соотношениям

$$
S_{3}^{\alpha}(t, y, a) \geqslant\left. S_{3}^{\alpha}\right|_{y_{2}=0} \equiv 0, \quad \min _{v \in M} B\left[S_{3}^{0}, v\right]=0 \text { на } P^{0} \times A,
$$
$\max _{z \in G} \min B\left[S_{3}^{1}, v\right]=0$ на $P^{0} \times A\left(\left.B\left[S_{3}^{\alpha}, v^{0}\right] \equiv \frac{d S^{\alpha}}{d t}\right|_{v=v^{0}}+L_{3}^{\prime}\left(v^{0}\right), \alpha=0,1\right)$,

$$
\begin{gathered}
v_{3}^{\alpha}=\left(z_{\beta}^{\alpha}(t, y, a), u_{\sigma 3}^{\alpha}(t, y, a)\right)^{*}, \\
J_{3}\left[v_{3}^{\alpha}\right]=S_{3}^{\alpha}, \quad v_{3}^{\alpha} \in M^{\prime}(\beta=\overline{1, n-2}, \sigma=\overline{1, r} \leqslant n),
\end{gathered}
$$

$M^{\prime}=\left\{v^{\prime}(t, y, a) \subset C, v^{\prime} \in M, y\left[t, P_{1}^{0} \mid v^{\prime}\right] \in P^{0}, \quad y_{2}\left[t_{1}, P_{1}^{0} \mid v^{\prime}\right]=0\left(t_{0} \leqslant t \leqslant t_{1}\right)\right.$,

$$
S_{3}^{0}(t, y, a) \leqslant S_{3}(t, y, z, a) \equiv
$$$$
\equiv \min _{u^{\prime \prime} \in U^{\prime \prime}} J_{3} \leqslant S_{3}^{1}(t, y, a)\left(D_{1}^{0} \times A \ni\left(y_{1}, y_{2}, z_{\beta}, a_{k}\right)^{*}, k \geqslant 1\right) \text {. }
$$

Последние неравенства являются следствиями используемого предположения о существовании достаточно гладкого оптимального решения $u_{0}^{\prime \prime}(t, \xi, a), S_{3}=J_{3}\left[u_{0}^{\prime \prime}\right]$ задачи (2.3) и уравнений (2.16), из которых они получаются интегрированием вдоль $(2.3) \quad\left(u \equiv \equiv u_{0}^{\prime \prime}\right)$ оценок на $D^{0} \times A$, соответствующих $(2.16)$

$$
\left(d S_{3}^{1} / d t\right)_{u=u_{0}^{\prime \prime}} \leqslant-L_{3}^{\prime}\left(t, \xi, u_{0}^{\prime \prime}, a\right) \leqslant\left(d S_{3}^{0} / d t\right)_{u=u_{0}^{\prime \prime}}(\xi=F(t, \xi, u, a)) .
$$

Неравенства очевидны геометрически при подстановке в показатели (2.15) допустимого для $\Sigma_{0}, \Sigma_{1}$ оптимального элемента ' $u_{0}^{\prime \prime}$, $\xi^{0}$ модели (2.3). Регуляторы $u_{3}{ }^{0}(t, y, a)$ и $u_{3}{ }^{1}(t, y, a)$ оптимальны для экстремальных агрегированных систем $\Sigma_{0}{ }^{*}$ и $\Sigma_{1}{ }^{*}$, полученных из $(2.15)$ заменой $z=z_{3}^{\alpha}(t, y, a) \quad\left(z_{3}^{\alpha}=\left(z_{\beta}{ }^{\alpha}\right)^{*}, \alpha=0,1, \beta=\overline{1, n-2)}\right.$

$d y^{0} d d t=Y\left(t, y^{0}, z^{0}\left(t, y^{0}, a\right), u, a\right), J_{3}^{* 0}=\int_{t}^{t_{1}^{0}} L_{3}^{\prime}\left(\tau, y^{0}, z^{0}\left(t, y^{0}, a\right), u, a\right) d \tau$,

$$
d y^{1} / d t=Y\left(t, y^{1}, z^{1}\left(t, y^{1}, a\right), u, a\right)
$$




$$
\begin{gathered}
J_{3}^{* 1}=\int_{t}^{t_{1}^{1}} L_{3}^{\prime}\left(\tau, y^{1}, z^{1}\left(t, y^{1}, a\right), u, a\right) d \tau\left(y_{2}^{\alpha}\left[t_{1}^{\alpha}\right]=0\right), \\
S_{3}^{\alpha}(t, y, a)=\min _{u \in U_{*}^{*}}^{* \alpha}
\end{gathered}
$$

$\left(\operatorname{dim} y=2, t_{0}, y_{0}^{\alpha} \in P_{1}^{0}, y^{\alpha}[t] \in P^{0}, t \leqslant t_{1}^{\alpha}, u_{3}^{\alpha} \in U_{*}^{\prime \prime}, \alpha=0,1\right)$.

Следовательно, они субоптимальны для (2.3) при $L^{\prime} \equiv L_{3}^{\prime}$. Допустим, что $u_{3}{ }^{0}, u_{3}{ }^{1}$ - регуляторы, стабилизирующие $(2.3)$ на $D_{1}{ }^{0}\left(u_{3}{ }^{\alpha} \in U^{\prime \prime}\right)$. Тогда аналогично $(2.7),(2.17)$ из (2.16) находим на $D_{1}^{0} \times A$ оценки

$$
\begin{gathered}
S_{3}^{0}(t, y, a) \leqslant J_{3}\left(t, \xi, a \mid u_{3}^{0}\right), S_{3}(t, \xi, a) \leqslant J_{3}\left(t, \xi, a \mid u_{3}^{1}\right), \\
J_{3}\left(t, \xi, a \mid u_{3}^{1}\right) \leqslant S_{3}^{1}(t, y, a),
\end{gathered}
$$

$$
J_{3}\left(t, \xi, a \mid u_{3}^{0}\right)-J_{3}\left(t, \xi, a \mid u_{3}^{1}\right) \geqslant S_{3}^{0}(t, y, a)-S_{3}^{1}(t, y, a) \quad(\alpha=0,1) .
$$

Обозначим через $t_{0}{ }^{0}, t_{0}{ }^{1}$ первые моменты $\lim y_{2}{ }^{\alpha}[t]=0$ при $t \rightarrow t_{0}{ }^{\alpha}$ соответственно для $u=u_{3}^{\alpha}$ в (2.3). Интегрируя $B\left[S_{3}{ }^{\alpha}, u_{3}{ }^{\delta}\right] \quad(\delta=0,1)$ вдоль (2.3) для $\forall t_{0}, \xi_{0} \in D_{1}{ }^{0}$, получим величину $\mathrm{Q}=2\left[J_{3}\left(t, \xi, a \mid u_{3}{ }^{0}\right)-\right.$ $\left.-J_{3}\left(t, \xi, a \mid u_{3}{ }^{1}\right)\right]$, равную выражению

$\varrho[t]=\int_{t}^{t_{0}^{0}}\left(B\left[S_{3}^{1}, u_{3}^{0}\right]+B\left[S_{3}^{0}, u_{3}^{0}\right]\right) d \tau-\int_{t}^{t_{0}^{1}}\left(B\left[S_{3}^{1}, u_{3}^{1}\right]+B\left[S_{3}^{0}, u_{3}^{1}\right]\right) d \tau$,

знак которой фиксирует выбор $u_{3}$ д для лучшего приближения решения задачи (2.3). Если $t_{0}{ }^{0}=t_{0}{ }^{1}$ и $\mathrm{Q}[t]$ монотонная функция, то для индекса $\alpha=0,1$ имеем

$$
2 \alpha=1+\operatorname{sign}\left(B\left[S_{3}^{1}, u_{3}^{0}\right]+B\left[S_{3}^{0}, u_{3}^{0}\right]-B\left[S_{3}^{1}, u_{3}^{1}\right]-B\left[S_{3}^{0}, u_{3}^{1}\right]\right) .
$$

Из-за сложности проверки $(2.20)$ в общем случае для субоптимальной стабилизации (2.3) выберем $u_{3}^{1}$ согласно (2.19). Критерии $a$-оптимизации приближения $S_{3}{ }^{1}, u_{3}^{1}$ получаем из (2.9) заменой $V_{+}{ }^{1}, V_{+}{ }^{(2)}$ на $S_{\alpha}{ }^{*}(t, x, a) \equiv S_{3}{ }^{\alpha}(t, y(t, x, a), a), I_{3}\left(t, x \mid u_{3}{ }^{\prime \alpha}\right) \quad\left(u^{\prime}=u(\xi(x))\right)$ с учетом обозначений $(2.3),(2.18)$. При неопределенности выбора $u_{3}^{\alpha}$ заменим $V_{+}{ }^{1}$ и $V_{+}{ }^{(2)}$ соответственно на $\left|I_{3}\left(u_{3}{ }^{1 \prime}\right)-I_{3}\left(u_{3}{ }^{{ }^{\prime}}\right)\right|$ и $\left|S_{\alpha}{ }^{*}-S_{\delta}{ }^{*}\right|(\alpha \neq$ $\neq \delta=0,1)$.

Из сравнения систем $(2.11),(2.18)$ следует, что в отличие от 2.2 в варианте 2.3 вектор-функция $z_{3}^{\alpha}=\gamma^{\alpha}(t, y, a)$ подчинена условиям (2.14), (2.16) экстремальной задачи (2.15).

2.4. $y$-А в тономны й $\left[{ }^{7,8}\right]$ случ у й задачи (2.3), (2.14). В этом варианте на $D^{0} \times A$ существует оптимальное решение $S^{0}(t, y, a)$, $u^{0}(t, \xi, a) \in U^{\prime \prime}$ задачи (2.3), удовлетворяющее условиям

$$
\begin{gathered}
S^{0} \geqslant\left. S^{0}\right|_{y_{2}=0} \equiv 0, B\left[S^{0}, u\right] \geqslant B\left[S^{0}, u^{0}\right] \equiv 0 \quad\left(t, y, a \in P \times A, u, u^{0} \in U^{\prime \prime}\right), \\
B\left[S^{*}, u^{*}\right] \equiv \lambda(t, \xi, a) b\left(t, y, p_{*}^{\prime}, a\right) \\
\left(S^{*}=S^{*}(t, y, a), S^{*}, S^{0} \subset C(P \times A), S^{*}, S^{0} \subset C_{1}\left(P^{0} \times A\right)\right), \\
b\left(t, y, p^{\prime}, a\right) \equiv 0, \partial b / \partial p^{\prime} \neq 0 \text { на } P^{0} \times A, \lambda \in C\left(D^{0} \times A\right) \\
\left(p^{\prime}=\left(\frac{\partial S^{*}}{\partial t}, \frac{\partial S^{*}}{\partial y_{\beta}}\right)^{*}, \beta=1,2\right),
\end{gathered}
$$


$B\left[S^{*}, u\right] \geqslant B\left[S^{*}, u^{*}\right], u^{*}=u^{*}\left(t, \xi, p_{*}, a\right), u^{0}=\left.u^{*}\right|_{S^{*}=S^{0}}\left(p_{*}=\left(\frac{\partial S^{*}}{\partial y_{\beta}}\right)^{*}\right)$.

Из (2.16) и (2.21) выводим, что $S_{3}{ }^{0}=S^{0}=S_{3}{ }^{1}$, если регуляторы

$u_{3}^{0} \equiv u^{*}\left(t, y, z^{0}, p_{*}, a\right)=u_{3}^{0}(t, y, a), u_{3}^{1} \equiv u^{*}\left(t, y, z^{1}, p_{*}, a\right)=u_{3}^{1}(t, y, a)$,

где $\lambda\left(t, y, z^{0}, a\right) \leqslant \lambda(t, y, z, a) \leqslant \lambda\left(t, y, z^{1}, a\right), z^{\alpha} \subset C\left(P^{0} \times A\right), \quad \alpha=0,1$, являются стабилизирующими на $D_{1}^{0}\left(u_{3}^{\alpha} \in U^{\prime \prime}\right)$ для (2.3). При этом условии из $(2.1),(2.19),(2.21)$ и $B\left[S^{0}, u_{3}^{\alpha}\right]=0$ получаем равенства

$$
S_{3}^{\alpha}=J_{3}\left(t, \xi, a \mid u_{3}^{\alpha}\right)=S^{0}(t, y, a)=\min _{u^{\prime \prime} \in U^{\prime \prime}} J_{3}\left(t, \xi, a \mid u^{\prime \prime}\right)=I_{3}^{0}(t, x)=\min _{u^{\prime} \in U^{\prime}} I_{3} .
$$

Отсюда заключаем, что экстремальные для (2.18) вектор-функции (2.22) будут оптимальными регуляторами задачи (2.3) ( $\left.L^{\prime} \equiv L_{3}^{\prime}\right)$, если система $(2.3),(2.14)$-автономна $(2.21)$ и $u_{3}^{\alpha} \in U^{\prime \prime}$. В силу независимости $I_{3}\left[u_{3}{ }^{\prime \alpha}\right]$ от $а$ при $u_{3}{ }^{\prime \alpha}=u_{3}{ }^{\prime \alpha}[t, y(x), a]$ оптимизируемая система является грубой и поэтому $а$-минимизация по (2.9) не требуется. Заметим, что экстремальные по (2.18) при $z=\gamma \cdot(t, y, a) \in G$ регуляторы $u_{3}\left[\gamma_{*}\right]=u^{*}\left(t, y, \gamma_{*}, p_{*}, a\right)$ оптимальны для задачи $(2.3)$, если $u_{3}\left[\gamma_{*}\right] \in$ $\in U^{\prime \prime}$. Для линейных по $u$ систем (1.1) предложены [ $\left.{ }^{7}\right]$ условия на матрицу, вводящую управление, и внутренняя аппроксимация нестационарных ограничений на $u$, при которых система (2.3) обращается в $y$-автономную.

3. Примеры. Применим результаты пп. 1 и 2 к задачам оптимальной стабилизации невозмущенного движения некоторых консервативных и $y$-автономных систем. Функции Беллмана и оптимальные регуляторы для этих классов систем удовлетворяют условиям теорем 1 и 2. Для $z$-стабилизации движения $z \equiv 0$ консервативной системы

$$
\begin{gathered}
q=\partial h / \partial p, p=A u-\partial h / \partial q\left(z=\left(q_{i}, p_{i}\right)^{*},\right. \\
q=\left(q_{i}\right)^{*}, p=\left(p_{i}\right)^{*}, u=\left(u_{s}\right)^{*}, i=\overline{1, n}, s=\overline{1, r)}, \\
h=h(z)>0, z \neq 0, h(0)=0,\left.\frac{\partial h}{\partial q}\right|_{p=0, q \neq 0} \neq 0 \\
\left(\frac{\partial h}{\partial z}=0, z=0, h \subset C_{2}(|z|<\infty)\right), \\
a^{1} \neq 0, p \neq 0 ; a^{1}=0, p=0 \\
\left(a^{1}=-A^{*} b, b=\frac{\partial h}{\partial p}, A=|p| A_{0}, A_{0}=\left\|a_{i s}(z)\right\| \subset C_{1}(|z|<\infty)\right)
\end{gathered}
$$

найдем при условии $0 \leqslant v(u) \leqslant v^{0}$, где $v(u)-$ положительно однородная выпуклая функция типа нормы $\left[{ }^{7}\right]$, регулятор $u^{0}$ и оптимальный по критерию функционал

$$
\begin{gathered}
I=\int_{t_{0}}^{t_{1}} \tau\left[f_{0}(h)+f_{1}(h, v)\right] d t\left(t_{1}=\infty, v^{0}=\text { const }>0, f_{0}, f_{1} \subset C(h \geqslant 0, v \geqslant 0)\right), \\
f_{1}(h, 0) \equiv 0,\left.\frac{\partial f_{1}}{\partial v}\right|_{v=0} \geqslant 0, \frac{\partial^{2} f_{1}}{\partial v^{2}}>0, f_{0} \geqslant v^{0} \frac{\partial f_{1}}{\partial v^{0}}-f_{1}\left(h, v^{0}\right) \equiv g_{1}(h)>0 \\
\tau=\tau(z)=\varrho\left(a^{1}\right), \varrho(u) \equiv \sup _{\Sigma}(u \cdot \xi) \text { на }
\end{gathered}
$$




$$
v(\xi)=1(\varrho(0)=0 ; \varrho>0, u \neq 0 ; r \leqslant n)
$$

на ограниченном множестве $N_{0}=\left\{z \mid h(z)<l^{0}\right\}$, где $0<l^{0}=$ const $<$ $<\sup h$ при $z \in E^{2 n}$. Множество $N_{0}$ ограничено, если $h \rightarrow \infty$ при $|z| \rightarrow \infty$. Функция $\mathrm{Q}(u)$ имеет все свойства сопряженной ей функции $v(u)\left[{ }^{7}\right]$. Экстремально решение

$$
\begin{gathered}
S_{1}=\int_{0}^{h} \psi(h) d h, 0 \leqslant h \leqslant l^{0}, u^{0}=v^{0} \partial \varrho / \partial a^{1}, z \in N_{0}^{0} \equiv N_{0} \backslash p=0, \\
v^{0} \psi(h)=f_{0}(h)+f_{1}\left(h, v^{0}\right) \\
\left(a^{1}=-A^{*} \partial h / \partial p ; a^{1} \neq 0 ; p \neq 0 ; u^{0}(z) \equiv 0, z \in N^{0} \backslash N_{0}^{0}\right) .
\end{gathered}
$$

В силу (3.1) при $u=u^{0}(\vec{z}), z \in N_{0}$, имеем неравенства

$$
\begin{gathered}
h=-v^{0} a^{1} \cdot \partial \mathrm{\varrho} / \partial a^{1}=-v^{0} \varrho\left(a^{1}\right) \leqslant 0, p^{\cdot}=-\partial h / \partial q \neq 0 \text { при } \\
p=0, z \neq 0\left(\varrho\left(a^{1}\right)>0, p \neq 0\right) .
\end{gathered}
$$

Отсюда по теореме Барбашина-Красовского находим, что регулятор (3.3) дает асимптотическую устойчивость $z \equiv 0$, если $z\left(t_{0}\right) \in N_{0}$. Тогда из теорем 1 и 2 следует, что $u^{0}$ - оптимальный по (3.2) регулятор задачи (3.1), (3.2). $S_{1}$ - минимум функционала $I(u)$ для $\forall z\left(t_{0}\right) \in N_{0}$. Регулятор $u^{0}(z)$ дает оптимальную стабилизацию $z \equiv 0$ в целом, если $h \rightarrow \infty$ при $|z| \rightarrow \infty$. Заменим равенства в (3.1) условиями

$$
\begin{gathered}
h_{1}(p) \leqslant h(z) \leqslant h_{2}(p), z \in C^{1}=\left\{z:|p| \leqslant c_{1}\right\} \\
\left(h_{\alpha}(0)=0, h_{\alpha} \subset C\left(C^{1}\right), c_{1}=\text { const, } \alpha=1,2\right),
\end{gathered}
$$

$\left|A^{*} \partial h / \partial p\right| \geqslant w(h)\left(w(0)=0, w(h) \subset C\left[0, l_{0}\right], \quad z \in C^{1}, \quad h(z) \leqslant l_{0} \equiv \sup _{z \in C^{1}} h\right)$.

Здесь равенство $A=|p| A_{0}$ не требуется, $h_{\alpha}(p), \omega(h) \gg 0$ на $C^{1},\left[0, l_{0}\right]$. Пусть $c_{0}=$ const - минимум модуля корня $h_{2}(p)=l_{1} \equiv \min h_{1}(p)$ при $|p|=c_{1}$. На $D^{1}=\left\{0 \leqslant h \leqslant l_{1}, z \in C^{1}\right\}$ рассмотрим

$$
\begin{gathered}
S_{1}^{0}(h)=v^{0^{-1}} \int_{0}^{h}\left[f_{0}(h)+f_{1}\left(h, v^{0}\right)\right] d h, 0 \leqslant h \leqslant l_{0}, u^{0}=v^{0} \frac{\partial Q}{\partial a^{1}} \\
\left(z \in C^{1}, p \neq 0, a^{1}=-A^{*} b \neq 0\right) .
\end{gathered}
$$

Из (3.2) - (3.5) получаем неравенства

$$
\begin{gathered}
\varrho\left(a^{1}\right) \geqslant \varrho_{0}\left|a^{1}\right| \geqslant \varrho_{0} w(h), d S_{1}^{0} / d t \leqslant-Q_{0}\left[f_{0}+f_{1}\left(h, v^{0}\right)\right] w(h), \\
B\left[S_{1}^{0}, u\right] \geqslant B\left[S_{1}^{0}, u^{0}\right]=0, .
\end{gathered}
$$

$\varrho_{0}=\min _{e} \varrho(e),|e|=1, u \in \mid\left\{v(u) \leqslant v^{0}\right\}, v\left(u^{0}\right)=v^{0}\left(z \in C^{1}, p \neq 0,0<h \leqslant l_{0}\right)$, при которых удовлетворяются условия теорем 1 и 2 , а $u^{0}(z)$ будет оптимальным по (3.2) регулятором р-стабилизации (3.1), (3.4) для любого $z\left(t_{0}\right) \in C^{0}=\left\{z:|p|<c_{0}, p \neq 0\right\}$. Здесь $t_{1} \leqslant \infty$, а переменная типа действия $y_{2}=h(z)-$ инвариант (3.1) при $u \equiv 0, \hat{b}=\partial h / \partial p$. стемы

Пусть для уравнений возмущенного движения регулируемой си-

$$
\begin{gathered}
x=X(t, x)+A(t, x) u\left(x=\left(x_{k}\right)^{*}, z=\left(t, x_{k}\right)^{*} \in E^{n+1},\right. \\
u=\left(u_{s}\right)^{*}, k=\overline{1, n}, s=\overline{1, r)},
\end{gathered}
$$

$0 \leqslant v(u) \leqslant v^{0}, X=\left(X_{k}\right)^{*}, X(t, 0) \equiv 0, A=\left\|a_{k s}\right\| \subset C_{1}\left(E^{n+1}\right), X \subset C_{1}\left(E^{n+1}\right)$, 


$$
x^{\prime}=\left(x_{i}\right)^{*}, x^{\prime \prime}=\left(x_{j}\right)^{*}, X^{1}=\left(X_{i}\right)^{*}, A_{1}=\left\|a_{i s}\right\|(i=\overline{1, \lambda} \leqslant n, j=\overline{\lambda+1, n} \geqslant r)
$$

известна $x^{\prime}$-определенно положительная функция $y(z)$ со свойствами

$$
\begin{gathered}
y_{1}\left(x^{\prime}\right) \leqslant y(z) \leqslant y_{2}\left(x^{\prime}\right) \\
\left(y_{\alpha}(0)=0, y_{\alpha} \rightarrow \infty,\left|x^{\prime}\right| \rightarrow \infty, y, y_{\alpha} \subset C\left(E^{n+1}\right), \alpha=1,2\right), \\
F[y] \equiv \tau g(y), x^{\prime} \neq 0 \\
\left(y \subset C_{1}\left(x^{\prime} \neq 0\right), \tau \equiv \varrho(-a), a \equiv A^{*} \frac{\partial y}{\partial x}, F=\frac{\partial}{\partial t}+X_{k} \frac{\partial}{\partial x_{k}}\right), \\
\sup _{y \rightarrow 0} g(y) \equiv g^{0}<v^{0},
\end{gathered}
$$

$\left|A^{*} \partial y / \partial x\right| \geqslant w(y)>0, x^{\prime} \neq 0, y \neq 0(w(0)=0, w \subset C(y \geqslant 0))$.

Здесь $y_{\alpha}\left(x^{\prime}\right), w(y)$ - положительно определенные функции на $E^{\lambda}$, $y \geqslant 0, g=g(y)$ непрерывна на $y>0$ и не зависит явно от $z, \varrho(u)$ задана формулой (3.2). Неравенства (3.7) справедливы для норм-инвариантной, а также $y$-автономной системы (3.6), удовлетворяющей условиям

$$
\begin{gathered}
y(z)=y^{1}\left(x^{\prime}\right) \equiv\left|P^{*} x^{\prime}\right|, P=\left\|p_{i s}\right\|=\text { const, } \operatorname{det} P \neq 0, \\
i, s=\overline{1, r}=\operatorname{dim} x^{\prime}=\operatorname{dim} u,
\end{gathered}
$$

$v(u)=v^{1}(u) \equiv\left|C^{*} u\right|, C=c_{0}^{-1} A_{1}^{*} P, A_{1}=\left\|a_{i s}\right\|=$ const, $\operatorname{det} A_{1} \neq 0, X^{1}=\left(X_{i}\right)^{*}$, $r \leqslant n$,

$\left|c_{0}\right|^{-1} P P^{*} x^{\prime} \cdot X^{1} \equiv g\left(y^{1}\right)\left|P^{*} x^{\prime}\right|, \quad\left|c_{0}\right| v^{0}>k^{0} p^{0}, \quad p^{0}=\max _{|e|=1}|P e|, \sup _{x^{\prime}=0, t \geqslant 0}\left|X^{1}\right| \equiv$ $\equiv k^{0}<\infty$,

при которых $\tau=\left|c_{0}\right|=$ const, $\quad v^{1}=\left|c_{0}\right|^{-1}\left|P^{*} A_{1} u\right|, \quad \mathrm{Q}^{1}(u)=\left|c_{0}\right| \times$ $\times\left|P^{-1} A_{1}^{*-1} u\right|$. Для $x^{\prime}$-стабилизации (3.6) найдем регулятор $u^{0}$, оптимальный по критерию

$$
\begin{gathered}
I=\int_{t_{0}}^{t_{1}} \tau(z)\left[k_{0}(y)+k_{1}(y) v(u)\right] d t\left(I^{1}=\left|c_{0}\right| \int_{t_{0}}^{t_{1}}\left[k_{0}\left(y^{1}\right)+k_{1}\left(y^{1}\right) v^{1}(u)\right] d t\right), \\
g(y) \geqslant l(y) \equiv-k_{0}(y) k_{1}^{-1}(y) \\
k_{0} \geqslant 0, k_{1}>0, y>0\left(k_{\gamma} \subset C(y \geqslant 0), t_{1} \leqslant \infty, \gamma=0,1\right)
\end{gathered}
$$

типа времени-импульса. Если существуют $y_{0}>0$, где $g\left(y_{0}\right) \geqslant v^{0}$, то обозначим через $d^{1}$ наименьший корень уравнения $g(y)=v^{0}$ и через $D_{0}{ }^{1}$ область в $E_{+}{ }^{n+2}$ вида $\left\{0<y<d^{1}, y(z)<d^{1}\right\}$. Из (3.6), (3.7), (3.9) находим выражения

$u^{0}(z)=v^{0} \partial_{\mathrm{Q}} / \partial a_{1}, \tau\left[k_{0}+v^{0} k_{1}+f_{2} \psi\right]=0,\left.y^{0}\right|_{u=u^{0}}=\tau f_{2}\left(f_{2} \equiv g-v^{0}, v\left(u^{0}\right)=v^{0}\right)$,

$$
\begin{gathered}
S=\int_{0}^{y} \psi(y) d y, 0 \leqslant y \leqslant d^{1}, \psi= \\
=-f_{2}^{-1}\left(k_{0}+v^{0} k_{1}\right), 0<y<d^{1}\left(a_{1}=-A^{*} \frac{\partial y}{\partial x}, \tau=\varrho\left(a_{1}\right)\right) .
\end{gathered}
$$

При условиях $k_{0} \equiv-g k_{1}, g \leqslant 0(y>0)$ получаем в $D_{0}{ }^{1}$ стационарный граничный режим $\left[{ }^{7}\right]$, для которого нмеем полную систему уравнений 


$$
\begin{gathered}
b^{(1)}\left[S_{*}\right]=b^{(2)}\left[S_{*}\right]=\left(b^{(1)}, b^{(2)}\right)= \\
=\frac{d g}{d y} b^{(2)}=0\left(b^{1}=k_{0}+g \psi_{*}, b^{(2)}=k_{1}-\psi_{*}, \psi=\frac{d S_{*}}{d y}\right),
\end{gathered}
$$

где

$$
\begin{aligned}
& g(y) \equiv l(y) \leqslant 0, y>0, u_{*}^{0}=u^{0}=v^{0} \partial_{\varrho} / \partial a_{1}, x^{\prime} \neq 0, \psi *=k_{1}(y), \\
& S_{*}=\left.S\right|_{l=g}=\int_{0}^{y} k_{1}(y) d y, y \geqslant 0\left(k_{0} k_{1}^{-1} \equiv-l, k_{1}, g \subset C_{1}(y \geqslant 0)\right) .
\end{aligned}
$$

При $g>l$ для $y>0$ на $D_{0}^{1}$ имеем нестационарный граничный режим и равенства $S^{*} \equiv S(y), 0 \leqslant y \leqslant d^{1}, u *^{1} \equiv u^{0}(z)$ на области $D_{0}{ }^{1}$. С учетом $(3.7)-(3.10)$ и неравенств $B[S, u] \geqslant B\left[S, u^{0}\right]=0, v\left(u^{0}\right)=v^{0}=$ $=$ const, $-S \cdot \geqslant Q_{0}\left(k_{0}+v^{0} k_{1}\right) w, Q_{0}=\min \varrho(e)(|e|=1)$ заключаем, что для всякого $z\left(t_{0}\right) \in C^{0}=\left\{t \geqslant 0,0<\left|x^{\prime}\right|<r_{\delta},\left|x^{\prime \prime}\right|<\infty\right\} \quad(\delta=0,1)$ регулятор (3.10) - оптимальный для (3.6), (3.7), (3.9). Здесь $r_{\delta}=$ const - минимальный модуль корня уравнения $y_{2}\left(x^{\prime}\right)=d^{\delta}$, $d^{0}=\min y_{1}\left(x^{\prime}\right)$ при $\left|x^{\prime}\right|=r_{1}=$ const. Регулятор (3.10) оптимально $x^{\prime}$-стабилизирует (3.6) в целом, если $f_{2}<0$ на $y>0$. Это справедливо в случае (3.11) для $\forall v^{0}>0$. При $I=I^{1}$ из (3.8), (3.10) находим простые выражения

$$
\begin{gathered}
u^{0}=-\left|c_{0}\right| v^{0} A_{1}^{-1} x^{\prime}\left|P^{*} x^{\prime}\right|^{-1}, \\
t_{1}=t_{0}+\left|c_{0}\right|^{-1} \int_{0}^{\nu_{0}^{1}}\left(v^{0}-g(\xi)\right)^{-1} d \xi<\infty\left(y^{1}\left(t_{0}\right)<d^{1}, y^{1}=\left|P^{*} x^{\prime}\right|\right),
\end{gathered}
$$

аналогичные формулам Атанса-Летова, полученным для случая

$$
\lambda=r=n, y^{1}=|x|, v^{1}(u)=|u|, g\left(y^{1}\right) \equiv 0, k_{\delta}=\text { const } \geqslant 0, \delta=0,1 .
$$

\section{Л ИТЕ Р А Т У Р А}

1. К р а совски й Н. Н. Проблемы стабилизации управляемых движений. - В кн.: Малкин И. Г. «Теория устойчивости движений», доп. 4. М., 1966, с. $475-514$.

2. Румянцев В. В. Об оптимальной стабилизации управляемых систем. -- ПММ, 1970 , т. 34, вып. 3, с. $440-456$

3. Д ем ин В. Г., Фу р асов В. Д. К стабилизашии vправляемых систем по части переменных. - ПММ. 1976. т. 47. вып. 2, с. 355-359.

4. Wismer, D. A., A oki. M. Optimization methods for large-scale systems, 5 agfregation. New York, 1971, p. 191-232.

5. Ульм С. Метпл агрегашин лля синтеза субпптимальных управлений. - Изв. АН ЭССР. Физ. Матем.. 1971. т. 90, № 1, г. 3-7.

6. Grujič, Lj. T., Ge nt ina, J. C.. B orne, P. General aggregation of large-scale svstems by vector Lvapunov functions and vector norms. - Int. J. Control, 1976. v. $24, \mathrm{~N} 4$, p. $529-537$.

7. Кей С И. О понижении размерности в залачах оптимального синтеза некоторых динамических систем. - Изв. АН ЭССР, Физ. Матем., 1977, т. 26, № 1, c. $37-47$.

8. Л етов А. М. Динамика полета и vправления. М., 1969, с. 130-174.

9. Kle inman, D. L.. A tha ns M. The design of suboptimal linear time-varving systems. - IEEE Trans. Automat. Control, 1968, v. AC-13, N 2, p. 150-152.

10. Ульм С. Ю. О ппиближенных методах синтеза управлений. - Автоматика и телемеханика, 1972 , № 5 , с. $27-32$.

Институт кибернетики

Академии наук Эстонской ССР
Поступила в редакшию 20/IX 1977 


\section{KEIS}

\section{JUHTIMISE SUBOPTIMAALSEST SUNTEESIST AGREGEERIMISMEETODIL}

Artiklis on vaadeldud mittelineaarse dünaamilise süsteemi juhtimise ülesannet integraalse efektiivsusindeksi korral ja uuritud $n$-mõõtmelise süsteemi agregeerimise erinevaid viise juhtimise optimaalse sünteesi ülesande ligikaudseks lahendamiseks. Suboptimaalsed regulaatorid on leitud agregeeritud süsteemist Bellmann-Ljapunovi taastavate funktsioonide meetodil. On esitatud ligikaudsete lahendite optimeerimise kriteeriumid agregeerimisvektori järgi ja näited mōnede konservatiivsete ja $y$-autonoomsete süsteemide liikumise optimaalse stabiliseerimise ülesannete lahendamiseks.

\section{KEIS}

\section{SYNTHESIS OF SUBOPTIMAL CONTROL VARIANTS VIA AGGREGATION METHOD}

The control problem of nonlinear dynamical system with an integral performance index is considered in the paper. Various aggregation ways for approximate control synthesis are investigated. Suboptimal controls are determined via Lyapunov-Bellman generating functions for aggregated systems. Exact optimal solutions for $y$-autonomous systems and some criteria for optimal aggregation vector choice are put forward. 